\title{
New Synthesis Route for PHD Polyols
}

\author{
Vladimir Peshkov, Rozeta Evtimova, Michael Herzog, Gerhard Behrendt
}

\section{Summary}

The synthesis of polyurea dispersion polyols (PHD) was developed using a new route via depolymerization of polyurethane polyureas by a mixture of at least two glycols in the presence of a consumable catalyst, i. e. a secondary aliphatic amine. On this way such polyols were produced with a particle size distribution with a maximum in the 120 to 400 nanometer region with hydroxyl numbers of 180 to $300 \mathrm{mg} \mathrm{KOH} / \mathrm{g}$ being optically clear and highly reactive. The exact adjustment of the ethylene oxide content of the reaction mixture together with that of the polyether polyol originally present in the polyurethane polyurea used in a predetermined range of surface tension as well as specific reaction conditions leads to these polyols which will give in turn polyurethanes with exceptional properties.

\section{Zusammenfassung}

Es wird eine neuer Weg für die Herstellung von Harnstoff Dispersions-Polyolen (PHD) über die Depolymerisation von Polyurethan-Polyharnstoffen unter Verwendung einer Mischung von zumindest zwei Glykolen und einem Katalysator, z. B. einem sekundären aliphatischen Amin, entwickelt. Auf diesem Weg wurden Polyole mit einem Maximum der Teilchengrößenverteilung im Bereich 120 bis 140 Nanometer mit Hydroxyl-Zahlen im Bereich 180 bis 300 mg KOH/g bei optischer Klarheit und hoher Reaktivität hergestellt. Bei genauer Abstimmung des Ethylenoxidgehalts der Reaktionsmischung auf den der Polyetherpolyole, die ursprünglich im Polyurethan-Polyharnstoff verwendet wurden, sowie durch Arbeiten sowohl in einem definierten Bereich der Oberflächenspannung wie auch der Reaktionsbedingungen werden die beschriebenen Polyole erhalten, die wiederum zu Polyurethanen mit außergewöhnlichen Eigenschaften umgesetzt werden können.

\section{Introduction}

Polyurea dispersion polyols (PHD or PIPA polyols) are presently prepared by the in situ polyaddition reaction of isocyanates and amines in propylene oxide based high molecular weight polyether polyols [Narayan 1992]. The reaction between the added amine, usually ethanolamine or hydrazine, and the isocyanate, in general toluylene diisocyanate (TDI) is performed in high speed mixers in a way that only the amine group reacts with the formation of urea groups. The hydroxyl groups do not take part in the reaction. By this process, it is attempted to obtain the highest possible solids load in the polyol at lowest viscosities and long shelf life [Rische 2004]. The particle size of the polyureas in the polyol is beyond $5 \mu \mathrm{m}$ resulting in typical dispersions. In this paper we report on a new way to obtain stable dispersions with oligourea particles in the nanometer range with a load of 10 to $20 \%$ by weight. As a general method a combined aminolysis/glycolysis of polyurethane polyureas as are present in high resiliency foams [Behrendt 1999] is used. In this method the glycol component is ad-justed with a balanced proportion of ethylene and propylene oxide units and specific reaction conditions are to be used.

\section{Materials and Methods}

In the synthesis of the PHD polyols diethylene glycol (DEG) and dipropylene gycol (DPG) received from Stockmeyer $\mathrm{GmbH}$ were used without further purification. Polypropylene glycols were a gift from BASF AG which is gratefully acknowledged. Polyethylene glycols 200, 400, or 600 were received from BASF AG and used without further purification but only dried over molecular sieves. Di-n-butyl amine reagent grade was obtained from BASF AG. HR foams were received as a gift 
from Fehrer AG of Markranstädt, Germany. The general synthesis route is as follows: Into a 1.51 four necked glass flask equipped with magnetic stirrer, nitrogen inlet, reflux condenser, and thermocouple with exterior electric heating mantle and magnetic stirrer unit the calculated amounts of glycols were placed adding $5 \%$ b. w. of the secondary amine. After heating up the mixture to a temperature of $180^{\circ} \mathrm{C}$ the foam flakes were introduced with stirring. After 0 , completing the addition the reaction mixture is stirred for another 30 minutes at $220^{\circ} \mathrm{C}$. The reaction mixture is then cooled to ambient temperature and used without further purification or other treatment steps. Hydroxyl numbers and amine numbers were determined according to standards [DIN 1971]. The viscosity was measured through the rotation and oscillation mode of a Rheostress ${ }^{\circledR} 300$ (Haake $\mathrm{GmbH}$ ) with the temperature controlled at $25^{\circ} \mathrm{C}$. Particle sizes were determined using the NANOPHOX ${ }^{\circledast}$ laser light scattering method.

\section{Results}

In a first series of experiments the composition of the solvolysis mixture was composed of DEG and DPG with other parameters kept constant. The ratio of DEG and DPG was systematically changed in steps of $10 \%$ (table 1). By these changes the ethylene oxide content of the polyol mixture was gradually increased while the surface tension of the mixture gradually decreased taking the ethylene oxide end block of the polyether polyol in the HR foam as constant with $13 \% \mathrm{~b}$. w. The surface tension, usually measured in aqueous solution, can also be used as a parameter to predict the solubility of urea structures. As is shown in figure 1 the viscosity, the particle size of the oligoureas in the polyol, and the stabil- ity changed dramatically as a result of increasing ethylene oxide content. When using 100\% DPG, which is the typically used solvolysis reagent [Tucker 1976], the particle size exceeds $10 \mu \mathrm{m}$ in average and the reaction mixture tends to become instable. With increasing the ethylene oxide content, i. e. increasing the portion of DEG, the stability improves and the particle size decreases reaching a minimum at 40 to $50 \%$ of DEG or with a total ethylene oxide content of the mixture of about $25 \%$ b. w. In this range the particle size was found to be in the range of 100 to $400 \mathrm{~nm}$ with a maximum of the particle size distribution curve at $180 \mathrm{~nm}$.

As is seen from table 1 with 0 or $10 \% \mathrm{~b}$. w. of DEG in the solvolysis mixture the polyurea particles in the particulate reaction mixture are in the range above $1 \mu \mathrm{m}$. They were actually found to be in the range from

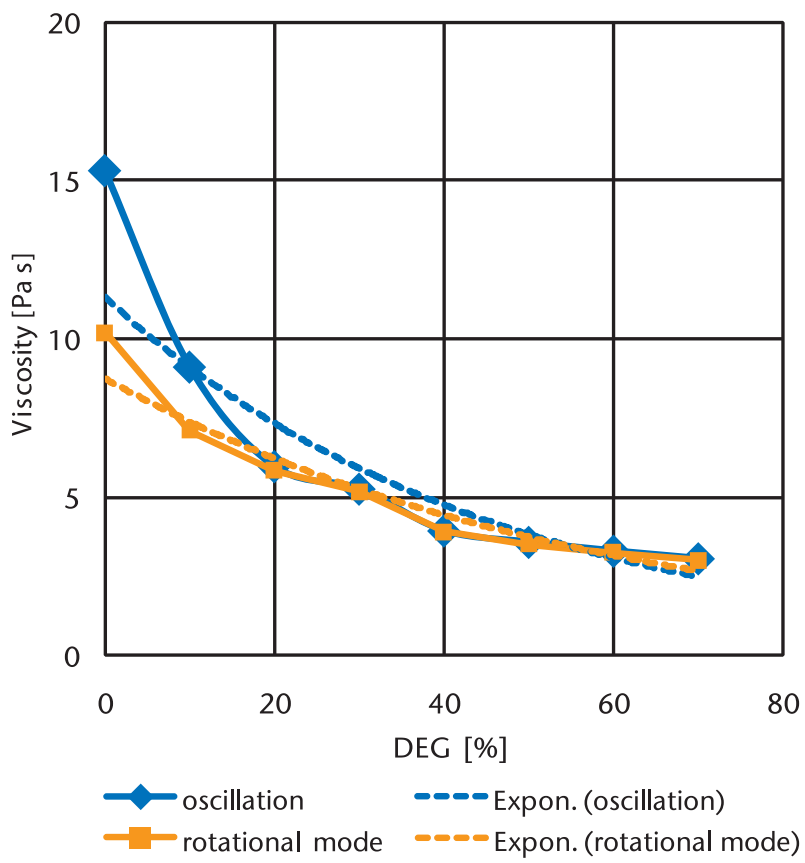

Fig. 1: Viscosity and stability of PHD polyols prepared from DEG/DPG mixtures as solvolysis reagent

\begin{tabular}{|l|l|l|l|l|l|l|l|l|l|}
\hline No. & 1.1 & 1.2 & 1.3 & 1.4 & 1.5 & 1.6 & 1.7 & 1.8 \\
\hline DPG:DEG & $100: 0$ & $90: 10$ & $80: 20$ & $70: 30$ & $60: 40$ & $50: 50$ & $40: 60$ & $30: 70$ \\
\hline Hydroxyl No. (mg KOH/g) & 280 & 302 & 310 & 320 & 333 & 344 & 330 & 340 \\
\hline Amine No. (mg KOH/g) & 58 & 58 & 56 & 58 & 59 & 57 & 55 & 59 \\
\hline Viscosity O (mPas) & 15,300 & 9,100 & 5,960 & 5,250 & 3,930 & 3,570 & 3,290 & $3,050 *$ ) \\
\hline Viscosity R (mPas) & 10,200 & 7,100 & 5,830 & 5,170 & 3,900 & 3,520 & 3,250 & $3,000 *$ \\
\hline Difference of viscosities & 5,100 & 2,000 & 130 & 80 & 30 & 50 & 40 & $-*$ ) \\
\hline Remarks & $\begin{array}{l}\text { particulate } \\
\text { dispersion }\end{array}$ & $\begin{array}{l}\text { particulate } \\
\text { dispersion }\end{array}$ & clear & clear & clear & clear & clear & two phases \\
\hline
\end{tabular}

Tab. 1: Solvolysis results of HR foams in DEG - DPG mixtures under constant conditions (63 \% b. w. foam, time of reaction 30 minutes, reaction temperature $220^{\circ} \mathrm{C}$ ) (viscosity measurement by oscillation »O«, by rotational mode »R«), *) The viscosity could be determined in the upper phase only so that no viscosity difference value is given. 


\begin{tabular}{|c|c|c|c|c|c|}
\hline No. & 17.5 & 17.4 & 17.1 & 17.6 & 17.7 \\
\hline HR foam (\%) & 53 & 58 & 63 & 68 & 73 \\
\hline Hydroxyl No. (mg KOH/g) & 400 & 338 & 330 & 248 & 202 \\
\hline Viscosity R (mPas) & 2,020 & 3,630 & 5,250 & 14,000 & 30,000 \\
\hline Viscosity O (mPas) & 2,080 & 3,700 & 5,290 & 16,700 & 42,000 \\
\hline Viscosity difference & 60 & 70 & 40 & 2,700 & 12,000 \\
\hline remarks & clear & clear & clear & turbid & turbid \\
\hline
\end{tabular}

Tab. 2: Effect of HR foam concentration on solvolysate properties using a ratio of DPG and DEG of 40 and 60

8 to $76 \mu \mathrm{m}$. With increasing the content of DEG in the solvolysis mixture the solvolysate becomes clear and the viscosity decreases to a minimum at $60 \%$ of DEG. Furthermore, the difference of viscosities determined in the rotational and oscillation mode also decreases to reach a minimum at a DEG content of $40 \%$ b. w. in the glycol mixture as well. This difference is a hint as to the type of viscosity, i. e. the higher this value the higher the proportion of non-Newtonian viscosity (structural viscosity) and, consequently, the particle size and the radii of solvation.

Polyols prepared by this method were stored at ambient temperature to check for their shelf life. It was observed that the shelf life increased with decreasing particle size reaching more than 360 days with the PHD polyols prepared with solvolysis glycol mixtures with between 40 and $60 \%$ b. w. of DEG. Further, the viscosity of the reaction mixture prepared at a reaction temperature at $180^{\circ} \mathrm{C}$ during addition and $220^{\circ} \mathrm{C}$ had its minimum at a ratio between 50 and $60 \%$ b. w. of DEG. It is assumed that in this range the stability is highest. The particle size distribution was measured by NANOPHOX and gave at $50 \%$ of DEG a distribution curve starting at $20 \mathrm{~nm}$ with a maximum of $120 \mathrm{~nm}$ and finishing at $320 \mathrm{~nm}$. It is suggested that for the lowest particle sizes of the oligoureas in the reaction mixture the viscosity itself and the difference between the viscosity as measured by rotational and oscillation mode has its minimum. At this, the stability of the polyols shows its maximum when using the same concentration of the oligoureas in the other mixtures of the series. The ef- fect of the concentration of the HR foam in the reaction mixture was derived in a series of experiments which data are shown in table 2 .

As can be seen from table 2 the optimum concentration of HR foam was found to be $63 \% \mathrm{~b}$. w. because at higher concentrations the viscosity of the reaction mixture rises sharply and becomes turbid due to larger particles. An increase of this concentration leads to a sharp increase in viscosity and especially in structural viscosity and, hence, to a tremendous increase in particle size giving rise to finally unstable and inhomogeneous solvolysates. By increasing the molecular weight of the polyethylene glycol (PEG) from 102 of DEG to 600 and using a similar approach with increasing the PEG content in the reaction mixture the results differed from those obtained with DEG with respect to stability and particle sizes (see table 3). When using PEG 200 (which is mainly tetraethylene glycol) instead of DEG and employing the same reaction conditions, solvolysis products could be obtained only in the range between 10 and $30 \%$ b. w. of PEG 200. Higher ratios always lead to unstable products and the formation of two phases. As the difference of the viscosities measured in the rotational and oscillation mode exceeded in all solvolysis products independent on the ethylene oxide content in the reaction mixture $700 \mathrm{mPas}$, it was assumed that the particle size was approaching $1 \mu \mathrm{m}$. This was verified by laser light scattering which found a distribution between $720 \mathrm{~nm}$ and $5.6 \mu \mathrm{m}$. Thus, it could be shown that PEG 200 was less suitable to be used as a solvolysis reagent due to the longer polyether chains and lower

\begin{tabular}{|c|c|c|c|c|c|}
\hline No. & 2.1 & 2.2 & 3.1 & 3.2 & 3.3 \\
\hline PEG 200 (\%) & 10 & 30 & 0 & 0 & 0 \\
\hline PEG $600(\%)$ & 0 & 0 & 10 & 30 & 50 \\
\hline Hydroxyl No. (mg KOH/g) & 322 & 284 & 284 & 238 & - \\
\hline Viscosity R (mPas) & 4,210 & 6,000 & 4,000 & 3,300 & - \\
\hline Viscosity O (mPas) & 4,890 & 6,880 & 4,220 & 3,330 & - \\
\hline Remarks & clear & slightly turbid & slightly turbid & slightly turbid & two phases \\
\hline
\end{tabular}

Tab. 3: Effect of higher molecular weight of polyethylene glycols used on the properties of solvolysis products (all other conditions as before) 


\begin{tabular}{|c|c|c|c|c|c|}
\hline No. & 7.1 & 7.2 & 7.3 & 7.4 & 7.5 \\
\hline MEG:PEG 200 & $90: 10$ & $70: 30$ & $50: 50$ & $70: 30$ & $10: 90$ \\
\hline Hydroxyl No. (mg KOH/g) & 345 & 314 & 268 & 212 & 159 \\
\hline Viscosity R (mPas)(1) & 2,560 & 2,450 & 2,240 & 2,340 & 1,930 \\
\hline Viscosity O (mPas)(1) & 2,620 & 2,380 & 2,300 & 2,300 & 1,970 \\
\hline Visosity difference & 60 & 70 & 60 & 40 & 40 \\
\hline Remarks & two phases & two phases & two phases & two phases & two phases \\
\hline
\end{tabular}

Tab. 4: Solvolysis of HR foams with mixtures of DPG and higher polyethylene glycols; (1) viscosities were measured in the top phase only

\begin{tabular}{|l|l|l|l|l|l|l|}
\hline No. & 5.1 & 5.2 & 5.3 & 5.4 & 5.5 & 5.6 \\
\hline DEG : PEG 200 & $90: 10$ & $80: 20$ & $70: 30$ & $60: 40$ & $50: 50$ & $30: 70$ \\
\hline Hydroxyl No. (mg KOH/g) & 262 & 225 & 213 & 200 & 184 & 159 \\
\hline Viscosity R (mPas) & 3,000 & 2,760 & 2,670 & 2,170 & 2,220 & 2,500 \\
\hline Viscosity O (mPas) & 3,050 & 2,800 & 2,700 & 2,200 & 2,250 & 2,56 \\
\hline Viscosity difference & 50 & 40 & 30 & 30 & 30 & 60 \\
\hline remarks & Top $80 \%$ & Top $70 \%$ & Top $70 \%$ & Top $65 \%$ & Top $60 \%$ & Top $70 \%$ \\
\hline
\end{tabular}

Tab. 5: Solvolysis of HR foams with DEG - PEG 200 mixtures (conditions as described, analytical values are for the top phase only)

hydroxyl group concentration. Using PEG 600 instead of PEG 200 and using up to $50 \%$ b. w. in the glycol mixture the results were similar. Only in the range of 10 to $25 \%$ b. w. of PEG 600 stable reaction products could be obtained. In contrast to the solvolysis products obtained with PEG 200 the differences in the measured viscosities were much smaller, i. e. in the range of 50 to $200 \mathrm{mPas}$. This hints to smaller particles in the solvolysis mixture. The values found were, nevertheless, in the same range as with PEG 200.

In another series, the dipropylene glycol was substituted by monoethylene glycol to avoid the effect of additional propylene units in the solvolysis mixture (table 4).

All solvolysates shown in table 4 resulted in two phase systems, the top phase being a clear viscous liquid while the bottom phase was a dark, turbid high viscous liquid. Such products are not useful in application and, consequently, were not further investigated. In another series of experiments the monoethylene glycol was substituted by DEG (table 5). The solvolysis products obtained with these mixtures were only two phase systems, i. e. the oligourea phase together with a minor amount of a glycol separated directly after synthesis. With increasing the amount of PEG 200 the amount of the top phase decreased to give a minimum at a 50:50 ratio of DEG : PEG 200. Thus, also such a combination of glycols for solvolysis of HR foams turned out to be of academic interest only.

In the series depicted in table 5, all top phases were clear viscous liquids while the bottom phases were dark, turbid, high viscous liquids. The process variant with these mixtures does neither lead to homogeneous solvolysis products nor to nanoscale oligoureas in them. Two other glycols were investigated to find further compositions to produce nanoscale oligourea dispersion polyols. In the first series of experiments the main component was chosen to be butane-1,4-diol (BD), used in admixture with various proportions with PEG 200. In any proportion between 10 and $50 \%$ b. w. of added PEG 200 the solvolysis products were again two phases and were not further analysed. In the second series of experiments a triple mixture of DPG, DEG, and polypropylene glycol of molecular weight 2000 (PPG 2000) was

\begin{tabular}{|c|c|c|c|c|}
\hline No. & 19.7 & 19.3 & 19.1 & 19.2 \\
\hline DPG : DEG : PPG & $40: 60: 0$ & $36: 54: 10$ & $32: 48: 20$ & $24: 36: 40$ \\
\hline Hydroxyl No. (mg KOH/g) & 330 & 290 & 275 & 217 \\
\hline Viscosity R (mPas) & 3,250 & 5,300 & 6,900 & 12,00 \\
\hline Viscosity O (mPas) & 3,290 & 6,400 & 8,900 & 13,600 \\
\hline Viscosity difference & 40 & 1,100 & 2,000 & 1,600 \\
\hline remarks & clear & clear & slightly turbid & turbid \\
\hline
\end{tabular}

Tab. 6: Solvolysis of HR foams with mixtures of DPG, DEG, and PPG 2000 
used (table 6). PPG 2000 was chosen so as to decrease the amount of short chain glycols which affect flexible foams produced from them in a negative way. In this series, under certain conditions clear, homogeneous solvolysis products containing nanoscale oligoureas were obtained.

Only at low concentrations in the range up to $15 \%$ b. w. of the long chain polyether diol stable homogeneous solvolysis products were obtained. But already the addition of $10 \% \mathrm{~b}$. w. of PPG 2000 to the solvolysis mixture results in an increase of the viscosity difference as an expression of increasing structural viscosity but the solvolysate remains clear showing that the particle size is below $1 \mu \mathrm{m}$. With increasing the percentage of PPG 2000 in the mixture the viscosity of the resultant polyol increases sharply and so does the difference of both viscosity measurement modes. This is again a hint to the effect of the ratio of ethylene oxide and propylene oxide but also on the effect of the amount of hydroxyl groups present in the mixture and the effect of longer polyether chains and coiling which may get into competition with the oligourea particles to either commingle with them or form complex adducts.

\section{Discussion of results}

HR foams are produced in general with a high molecular weight polyether polyol of a molecular mass of about 5000 to $5500 \mathrm{~g} / \mathrm{Mol}$ and an ethylene end block of about $13 \%$ b. w. as the basic component. Foaming is achieved by the reaction of water and an aromatic polyisocyanat, generally polymeric 4,4'-diphenylmethane diisocyanate (p-MDI). By using a molar excess of water, and its hydroxyl groups to the hydroxyl groups of the polyether alcohol in the range of 4 to 1 , the ratio of urea groups to urethane groups is in a similar ratio. The most simple molecule being formed would be 4,4'-diphenylmethane diamine (MDA) which has a molecular size in the range of $1.5 \mathrm{~nm}$ [Swardstrom 1972]. When only one urea group in a series based on MDA is present the size of the molecule exceeds $5 \mathrm{~nm}$. As a consequence of the formulation and the reactions proceeding during foaming, the distribution of the oligoureas is between 1 and 10 repeating units with a maximum of between 4 and 5 MDI residues, i. e. the average molecular size is in the range of 20 to $30 \mathrm{~nm}$. Consequently, shorter molecules or smaller particle sizes cannot be found. The particle size of the ureas as received from the foam should be - without considering agglomeration-in the range of 20 to $80 \mathrm{~nm}$ [Armistead 1988].

Urea groups form strong hydrogen bonds among themselves and to other groups being able to interact. Thus, the agglomeration of the urea groups during the foaming reaction and, further, during the solvolysis is the reason for larger particles and the particle size distribution found. When urea groups are agglomerated once, severe reaction conditions have to be used to split the hydrogen bonds. In such cases a side reaction occurs with cleavage of some of the urea groups in the end position of the blocks leading to formation of MDA. But, MDA is a carcerogen and, hence, has to be avoided so as to minimise problems with legislation. The increasing amount of MDA is found when increasing both the reaction time and temperature. MDA has another undesired effect - it leads to increases in the viscosity of the mixture due to thixotroping.

By careful adjustment of the reaction conditions and the use of increased ethylene oxide content in the solvolysis mixture both the formation of MDA and the agglomeration of the urea groups can be reduced. Hence, the tendency for oligoureas to form larger structures can be minimized leading to low viscosity and long time stable nanoscale oligourea dispersion polyols [Peshkov 2008]. By careful investigation of several types of glycols, reaction conditions, and percentage of HR foams in the reaction mixture, it was shown that only with a proportion of the short chain glycols DEG and DPG of about equal amounts stable PHD polyols could be produced. The ratio of DPG and DEG to produce stable PHD polyols with the oligoureas in the nanometer range was shown to be between $55 \%$ and $40 \% \mathrm{~b}$. w. of DPG. Homogeneous, clear polyols with a percentage of nanoscale oligoureas between 10 and $20 \%$ b. w. showing a particle size in the region starting with $20 \mathrm{~nm}$ in the NANOPHOX curve could be obtained only by using simple mixtures of DEG and DPG in a certain proportion. The use of monoethylene glycol, butane-1,4-diol, or higher polyethylene glycols had only a negative effect and resulted in two phase systems.

Polyetherpolyols build of $\mathrm{C}_{2-5}$ alkoxides are suitable to stabilize nanoscale structures particularly by reduction of the surface energy. Typical water-based microemulsions show spontaneously formed droplets in the range of some $10 \mathrm{~nm}$ to $100 \mathrm{~nm}$. For the purpose of stabilizing microemulsions a variety of alkoxy derivates with detergent properties is available with the Lutensol ${ }^{\circledR}$ product group of the BASF AG [Nguyen-Kim 2006]. Our 
experiments have shown that not only nanostructures in liquid aqueous systems may be stabilized but also dispersions of solid particles are formed and stabilized by appropriate polyether systems. The PHD polyols thus produced are suitable for the production of flexible, semi flexible, and rigid polyurethanes, especially coatings. These polyols when reacted with suitable diisocyanates or polyisocyanates result in polyurethanes with extraordinary properties [Peshkov 2006]. If such two component systems composed of the stable PHD polyols and suitable polyisocyanates are reacted in the absence of foaming agents under film forming conditions, coatings with exceptional high mechanical and thermo-mechanical properties are obtained [Peshkov 2010] which maybe used as leather, textile or metal coatings.

\section{Acknowledgements}

V.P. gratefully acknowledges support of Lausitzer Edelstahltechnik GmbH for financial support. The authors gratefully acknowledge gifts of polyethers from BASF AG.

\section{References}

[Narayan 1992] Narayan, T., Kinnaird, M.G. (BASF Corp.): Polyurea Based Dispersions, Foams Prepared Therefrom, and a Process for the Preparation Therein, USP 5,288,766 (28.02.1992)

[Rische 2004] Rische, T., Naujocks, K., Feller, T., Gurtler, C., Mazaneck, J. (Bayer AG): Polyurethane Polyurea Dispersions, USP 6,784,243 (31.08.2004)

[Behrendt 1999] Behrendt, G., Pohl, M.: Verfahren zur Herstellung von Polyolen und diese Polyole, DE-OS 19917932 (21.10.1999), EP1086169, USP 6.683.119 (27.01.2004)

[DIN 1971] DIN 53240: Bestimmung der Hydroxylzahl, 1971-12

[Tucker 1976] Tucker, B., Ulrich, H. (Upjohn Co.): Novel Process of Reclaiming Polyurethane Foam, USP 3,983,087 (28.09.1976)

[Swardstrom 1972] Swardstrom, J.W., Duvall, L.A., Miller, D.P.: The Crystal and Molecular Structure of Tris-(4,4' diaminodiphenylmethane)-Sodium Chloride, Acta Cryst. B28, 2510-2514 (1972)

[Armistead 1988] Armistead, J.P., Wilkes, G.L., Turner, R.B.: J. Appl. Polym. Sci. 35, 601629 (1988); Hespe, H., Meisert, E., Eisele, U., Morbitzer, L., Goyert, W.: Kolloid Z. und Polym. 250, 797 (1972)

[Peshkov 2008] Peshkov, V., Hunger, H.-D., Behrendt, G. (Technische Fachhochschule Wildau): Neue Polyharnstoff-Dispersionspolyole und Verfahren zu ihrer Herstellung, DE-Pat.-Anm. 102008008 $045.4(05.02 .2008)$

[Nguyen-Kim 2006] Nguyen-Kim, S. (BASF AG), Urethane Compound Comprising an Incorporated Polyether Group-Containing Silicone Derivative an a Nitrogen Heterocycle, WO 2006/069742 A1 (06.07.2006)

[Peshkov 2006] Peshkov, V., Naber, B.W., Schmidt, K.-H., Behrendt, G. (Recyclit GmbH): Verfahren zur Herstellung neuer, flammgeschützter Polyurethane, DE-Anm. 102006058400.7 (07.12.2006)

[Peshkov 2010] Peshkov, V., Knoll, U., Evtimova, R., Behrendt, G.: Polyurethanes Based on New PHD polyols, J. Univ. Chem. Technol. Metallurgy, in preparation

\section{Autors}

Dipl.-Ing. Vladimir Peshkov

University of Chemical Technology and Metallurgy

8 Kl. Ohridski bul. 1756 Sofia, Bulgaria

peshkov@uctm.edu

Prof. Dr. Rozeta Evtimova

University of Chemical Technology and Metallurgy

8 Kl. Ohridski bul. 1756 Sofia, Bulgaria

evtimova@uctm.edu

Dr. rer. nat. Michael Herzog

Fachbereich Ingenieurwesen/Wirtschaftsingenieurwesen

Technische Hochschule Wildau [FH]

michael.herzog@th-wildau.de

www.th-wildau.de/mherzog

Prof. Dr. rer. nat. Gerhard Behrendt

Fachbereich Ingenieurwesen/Wirtschaftsingenieurwesen

Technische Hochschule Wildau [FH]

gerhard.behrendt@th-wildau.de 